\title{
Total neutron cross-section extracted from transmission experiments with liquid oxygen using neutron energies from 18 to $27 \mathrm{MeV}$
}

\author{
Martin Ansorge ${ }^{1,2, *}$, Pavel Bém ${ }^{1}$, David Hladík ${ }^{1}$, Mitja Majerle $^{1}$, Jaromír Mrázek ${ }^{1}$, Jan Novák ${ }^{1}$, Eva Šimečková ${ }^{1}$, and \\ Milan Štefánik ${ }^{1,2}$ \\ ${ }^{1}$ Nuclear Physics Institute of the Czech Academy of Sciences, 25068 Řež, Czech Republic \\ ${ }^{2}$ Faculty of Nuclear Sciences and Physical Engineering, Czech Technical University in Prague, Prague 8, Czech Republic
}

\begin{abstract}
Fast neutron generators driven by isochronous cyclotron U-120M developed at Nuclear Physics Institute were used for neutron cross-section measurements. The target station with $2 \mathrm{~mm}$ thin lithium was used to produce pulsed beams of fast quasi-monoenergetic neutrons in the ${ }^{7} \operatorname{Li}(\mathrm{p}, \mathrm{n})$ reaction. The Time-ofFlight technique was used to determine the exact neutron energy spectra. The transmission measurements on liquid oxygen were conducted for several neutron peak energies between 18 and $27 \mathrm{MeV}$ and the cross-sections for reaction ${ }^{\text {nat }} \mathrm{O}(\mathrm{n}, \mathrm{tot})$ were derived.
\end{abstract}

\section{Introduction}

The total cross-section ${ }^{\text {nat }} \mathrm{O}(\mathrm{n}, \mathrm{tot})$ for reactions induced by fast neutrons is an important quantity for designing future energy facilities, where high fluxes of fast neutrons will be present together with sufficient amount of oxygen nuclei. In the energy region from 18 to $35 \mathrm{MeV}$ precise experimental data are still rare, making space for new additional transmission experiments with oxygen nuclei. Present results should improve the correctness of data evaluations and contribute to all projects and researches where crosssection ${ }^{n a t} \mathrm{O}(\mathrm{n}$,tot $)$ is not a negligible factor. The Accelerator Driven Systems (ADS) and future fusion-based facilities can be stated as relevant examples.

In recent years a series of transmission experiments has been conducted at the Nuclear Physics Institute (NPI) of the CAS at U-120M cyclotron facility, operated with the support of CANAM project. Fast neutron generators based on the interaction of protons with thin ${ }^{7} \mathrm{Li}$ were used together with Time-Of-Flight (TOF) technique based detection method.

\section{Experimental arrangement}

The production of fast neutrons at the NPI was carried out by U-120M cyclotron driven neutron source based on $\mathrm{p}+{ }^{7} \mathrm{Li}$ reaction. Target station with $2 \mathrm{~mm}$ thin $\mathrm{Li}$ foil (backed with thick graphite beam stopper) was used to produce neutrons with quasi-monoenergetic (QM) spectra. During four dedicated experiments, the U-120M cyclotron was set to accelerate primary protons at four different energies ranging from $20 \mathrm{MeV}$ to $35 \mathrm{MeV}$. A detailed description of the NPI neutron sources can be found in reference [1].

\footnotetext{
*e-mail: ansorge@ujf.cas.cz
}

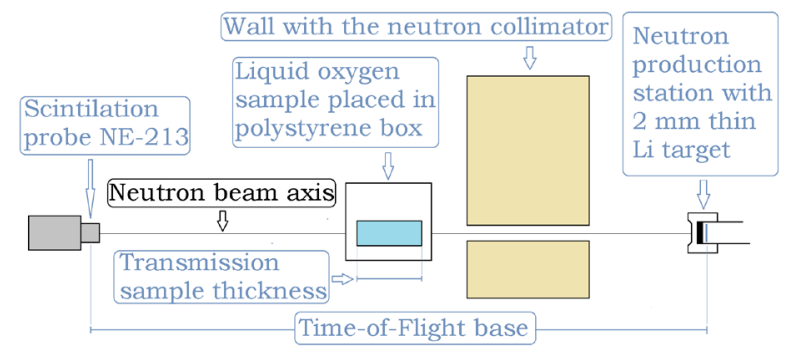

Figure 1. The basic scheme of an experimental setup.

The scheme of the basic geometry setup is shown in Fig. 1. During all measurements, the transmission sample was placed into the axis of the collimated neutron beam. The laser beam was used for the sample alignment into the neutron beam axis. Flight paths ranged from $9 \mathrm{~m}$ to $9.5 \mathrm{~m}$. The collimator was $1.6 \mathrm{~m}$ long with the diameter of $3 \mathrm{~cm}$. The setup with the collimator opened to $9 \mathrm{~cm}$ in diameter was used only for measurement with the kinetic energy of $27 \mathrm{MeV}$. In Fig. 2 the experimental setup behind the collimator is shown.

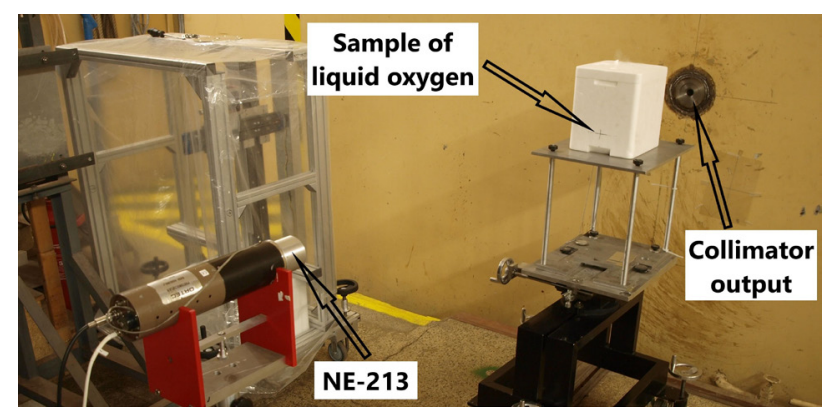

Figure 2. The experimental arrangement behind the collimator. 
The liquid oxygen used for measurements was of natural abundance and its volume purity was $99.95 \%$. During the measurements, the liquid oxygen was placed into a polystyrene (PS) box which can be seen in Fig. 2. The inner dimensions of the box base were $210 \mathrm{~mm} \times 160 \mathrm{~mm}$ at room temperature approximately. Exact dimensions were measured in the cooled state where PS box was filled with liquid nitrogen. Exact values are stated in Tab. 1 where std. deviations were determined as $\frac{0.2}{\sqrt{3}} \mathrm{~cm}$. The PS box was always placed during the experiments in the way that the liquid oxygen inside has shaded the active volume of the NE-213 scintillator.

The organic liquid scintillator NE-213 (cylindrical, $2 \times 2$ inches) coupled with CAEN digitizer V1751 (1000 $\mathrm{MS} / \mathrm{s}, 10 \mathrm{bit}$ ) was used for neutron detection and data acquisition. Neutron energy spectra were obtained by the TOF technique which was developed and successfully used in recent years at the NPI [2], [3]. Hereby, just a brief description of the TOF method will be given.

\section{Methodology}

For each kinetic energy of neutrons, the amount of detected neutrons $N_{\text {sample }}$ after transmission through a sample is measured and divided by the number of neutrons $N_{\text {freePath }}$ measured at the same energy and arrangement but without the sample placed in between the neutron source and the probe. Fraction $T_{l O 2}=\frac{N_{\text {sample }}}{N_{\text {freepath }}}$ is called transmission. Measured transmissions $T_{l O 2}$ were converted into cross sections $\sigma_{l O 2}$ by the familiar relationship:

$$
\sigma_{l O 2}=-\frac{1}{n l} \ln T_{l O 2},
$$

where $n$ is the number of atoms per unit volume and $l$ is the thickness of the liquid oxygen layer.

In the following subsections, the corrections resulting from the experimental setup will be described as well as the evaluation of overall uncertainties.

\subsection{Time-Of-Flight method}

During the TOF measurements, the digitizer records simultaneously the signal from the NE-213 probe and the signal of a cyclotron accelerating frequency. Cyclotron frequency is bounded to the timing of accelerated bunches of protons and it's used to derive the start signal for each detected pulse. The stop signal is taken directly from the NE-213 probe. Having a start and a stop timestamp a time of flight is calculated for each pulse. Subsequently, the kinetic energy is calculated for each neutron pulse detected. During neutron production in the target station also $\gamma$-particles with specific energies are produced especially in the interaction of protons with the graphite beam stopper. Since the NE-213 detector measured in mixed fields of neutron and $\gamma$ particles, the charge-comparison pulse shape discrimination method was used to distinguish neutron pulses from $\gamma$-pulses. In Fig. 3, the graph with neutron pulses plotted with respect to their amplitude (y-axis) and time of flight ( $\mathrm{x}$-axis) is shown. The range of the horizontal axis (in this case $49 \mathrm{~ns}$ ) is the period between two consecutive bunches of accelerated protons. Slicing the $2 \mathrm{D}$ representation as indicated in the Fig. 3, the experimental response function of the scintillator is obtained for the specific TOF and neutron kinetic energy respectively. The energy calibration of the probe is then derived from the experimental response functions. Red line outlines a situation where the whole kinetic energy of the detected neutron $E_{k i n_{n}}$ was absorbed by scattered hydrogen nucleus $E_{p_{\max }}$. Green line represents a situation where neutron transmits just a part of its kinetic energy $E_{p_{\min }}$ corresponding to the specific angle of $(n, p)$ interaction in the active volume of the scintillator. Final spectral points of the measured neutron energy spectrum are obtained by summing up the pulses in-between the green and red borders and subsequently by dividing with detector efficiencies calculated for the energy equivalent green-to-red region.

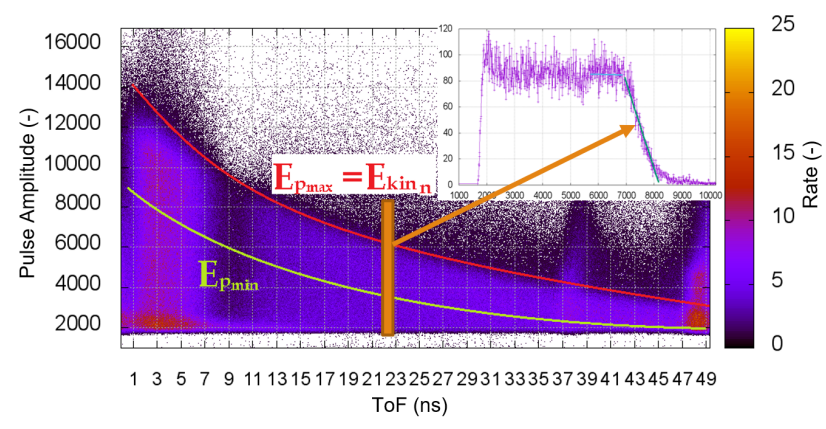

Figure 3. Example of the TOF spectrum of neutron pulses from Quasi-monoenergetic source and it's decomposition to particular time of flight.

Two-dimensional TOF spectrum depicted in Fig. 3 is an ideal example of measurement where the accelerated beam bunches are normally distributed in time with FWHM up to $1 \mathrm{~ns}$.
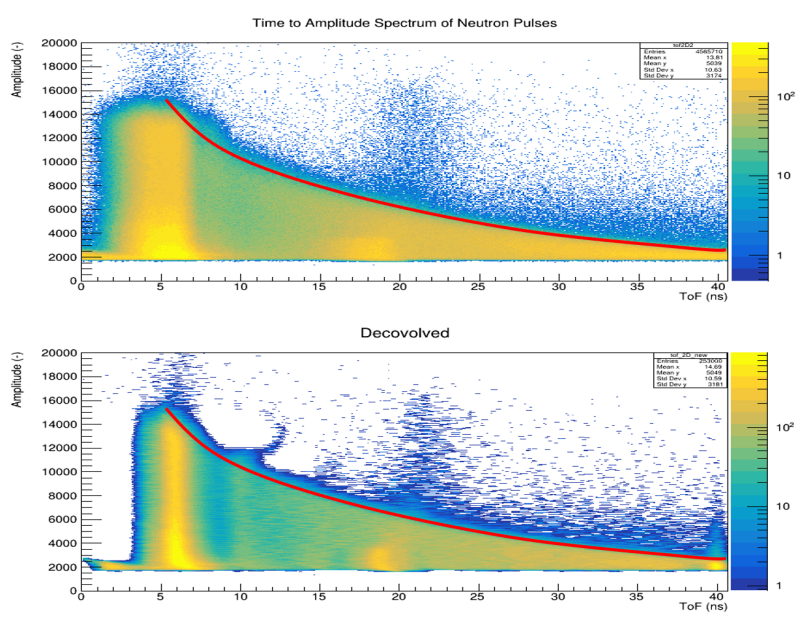

Figure 4. Example of the TOF spectrum where QM-peak is spread due to the non-gaussian time distribution of accelerated beam (upper). Enhancement of TOF spectrum time resolution with deconvolution (lower). 
Deconvolution of 2D TOF spectra was performed in cases where cyclotron exhibited non-gaussian time distribution of proton beams. The example of such improvement in time resolution is shown in Fig. 4. Improvement is most clearly visible in the region of the QM-peak which is also the most important region for our transmission experiments with the QM fast neutron source. Deconvolution was performed with internal ROOT-Framework Gold deconvolution method [4],[5]. The time distribution of detected $\gamma$-pulses was used as the response function for the deconvolution algorithm.

\subsection{Corrections}

With the described experimental setup (Sec. 2) and TOF technique (Sec. 3.1) QM neutron spectra were measured and integrals of QM-peaks were used to calculate the transmission $T_{l O 2}$. The following corrections were taken into account.

\subsubsection{Dead-Time}

With the data acquisition carried out by CAEN V1751 digitizer, only the time when data transfer from the digitizer to computer collapses (buffer overflow) is considered to be a dead time (DT). For a sufficiently low detector load, the DT was zero. However, in certain measurements, higher fluxes weren't avoided and DT correction had to be used. Particular values were determined directly from the saved data.

\subsubsection{Pile-Up}

The typical recorded sequence of the signal is $240 \mathrm{~ns}$ long for each pulse. If a new pulse occurs in the detector in those $240 \mathrm{~ns}$ then it is excluded from the final statistic. Pile-Up corrections were derived from the distribution of time differences between two consecutive pulses for each measurement. Depending on actual particle fluxes during irradiations this correction was of the order of 1 to $4 \%$ with that of $12 \%$ for the neutron energy of $22 \mathrm{MeV}$.

\subsubsection{Air displacement}

All the measurements were conducted in the presence of air. While removing the sample out of the beam it's volume is replaced with the air which should be taken into account. The loss of neutrons from the beam due to the transmission through the air can be calculated with the knowledge of total neutron cross-section for air [6] using the inverse formula to equation 1 . This correction was of the order of $0.15 \%$ for both orientations of the PS box.

\subsubsection{MCNPX correction of results}

The influence of the PS box and the "In-DetectorScattered" (IDS) effect were estimated by MC simulations. To investigate these effects the MCNPX 2.7.0 computational code [7] was used together with the ENDF/B-VII.1 data library [8]. For the IDS effect, the estimation of the fraction of events where neutron undergoes the interaction of any kind and still reaches the active volume of the simulated detector with sufficient kinetic energy was performed for each experimental setup. The assumption of neutrons uniformly distributed over the collimator crosssection was used for the definition of the source. The presence of the PS box causes the underestimation of the transmission results, approx. $1.5 \%$ of peak neutrons are lost from the beam due to the polystyrene (depending on the kinetic energy). In-Detector-Scattered effect causes the overestimation of the transmission results, corrections range from 0.2 to $1 \%$ (depending on kinetic energy and box orientation). Combining both corrections results into transmission values being underestimated from $0.2 \%$ to $1.3 \%$ at the energy range $18-27 \mathrm{MeV}$. Particular values of MCNPX corrections are tabulated in Tab. 1.

\subsection{Uncertainties}

TOF method had the major contribution to the final uncertainty of the total neutron CS values. The main source of the uncertainties of the TOF technique arises from the time resolution of the accelerated proton bunches and consequently from the energy calibration of the NE-213 probe. Systematic uncertainty of the TOF technique was investigated by the sensitivity analyses where the input parameters for the TOF method were sampled accordingly to its assumed std. deviation and probability distribution. Uncertainties due to the counting statistics were one order of magnitude lower compared to the systematic uncertainty of the TOF method. Both systematic and statistical uncertainties are combined as uncorrelated and are listed in the Tab. 1 as the standard deviation of uncorrected transmission values. Other minor uncertainties have been also taken into account - uncertainty in the length of the sample and uncertainties of the MCNPX and Air displacement corrections. Error approximation for both corrections stated in the Tab. 1 have been estimated conservatively as $20 \%$ of the correction values.

The uncertainties of the mean neutron energy of a QM-peaks were determined by the uncertainty analysis of the TOF method, see Tab. 1. The FWHM values are listed for each neutron energy as well. The influence of the QM-peak width on the final cross-section values has not been further investigated. According to the consideration made here [9], this effect is assumed to be negligible.

\section{Results}

The measured total neutron cross-section values for reaction ${ }^{\text {nat }} \mathrm{O}(\mathrm{n}$, tot $)$ are tabulated in Tab. 1. For the neutron energies $18.8 \mathrm{MeV}, 25 \mathrm{MeV}$ and $27 \mathrm{MeV}$ final results were calculated as the average of the two measurements (each measurement with different orientation of PS box). For the neutron energy $22 \mathrm{MeV}$ the measurement with only one orientation of the PS box was performed. Slight corrections have been applied to obtain the final results and the uncertainties have been estimated, for details see Sections 
Table 1. Results of transmission experiments with fast neutrons on liquid oxygen and exact values of corrections and uncertainties.

\begin{tabular}{cccccccc}
\hline $\begin{array}{c}\text { Mean Energy of } \\
\text { QM-peak (MeV) }\end{array}$ & $\begin{array}{c}\text { QM-peak } \\
\text { FWHM (MeV) }\end{array}$ & $\begin{array}{c}\text { Sample } \\
\text { thickness } l(\mathrm{~cm})\end{array}$ & $\begin{array}{c}\text { Uncorrected } \\
\text { transmission (-) }\end{array}$ & $\begin{array}{c}\text { Uncorrected } \\
\text { cross-section (b) }\end{array}$ & MCNPX corr. (b) & Air corr. (b) & $\begin{array}{c}\text { Corrected } \\
\text { average CS (b) }\end{array}$ \\
\hline \hline \multirow{2}{*}{$18.8 \pm 0.3$} & \multirow{2}{*}{$1.97 \pm 0.07$} & $20.80 \pm 0.12$ & $0.2345 \pm 0.0130$ & 1.622 & $-0.0087 \pm 0.018$ & $0.0024 \pm 0.0005$ & $1.622 \pm 0.044$ \\
& $15.88 \pm 0.12$ & $0.3266 \pm 0.0139$ & 1.640 & $-0.0151 \pm 0.031$ & $0.0026 \pm 0.0005$ & \\
\hline $22.0 \pm 0.2$ & $1.89 \pm 0.04$ & $20.80 \pm 0.12$ & $0.2227 \pm 0.0053$ & 1.680 & $-0.0143 \pm 0.029$ & $0.0023 \pm 0.0005$ & $1.668 \pm 0.028$ \\
\hline \multirow{2}{*}{$25.0 \pm 0.2$} & \multirow{2}{*}{$1.73 \pm 0.05$} & $20.80 \pm 0.12$ & $0.2359 \pm 0.0064$ & 1.616 & $-0.0047 \pm 0.011$ & $0.0023 \pm 0.0005$ & \multirow{2}{*}{$1.620 \pm 0.027$} \\
& & $15.88 \pm 0.12$ & $0.3276 \pm 0.0094$ & 1.635 & $-0.0110 \pm 0.023$ & $0.0024 \pm 0.0005$ & \\
\hline \multirow{2}{*}{$27.0 \pm 0.2$} & \multirow{2}{*}{$1.3 \pm 0.01$} & $20.80 \pm 0.12$ & $0.2399 \pm 0.0054$ & 1.597 & $-0.0109 \pm 0.022$ & $0.0023 \pm 0.0005$ & \multirow{2}{*}{$1.600 \pm 0.022$} \\
& & $15.88 \pm 0.12$ & $0.3296 \pm 0.0077$ & 1.626 & $-0.0164 \pm 0.033$ & $0.0024 \pm 0.0005$ & \\
\hline
\end{tabular}

3.2 and 3.3 respectively. Graphical representation of the present experimental data is shown in Fig. 5.

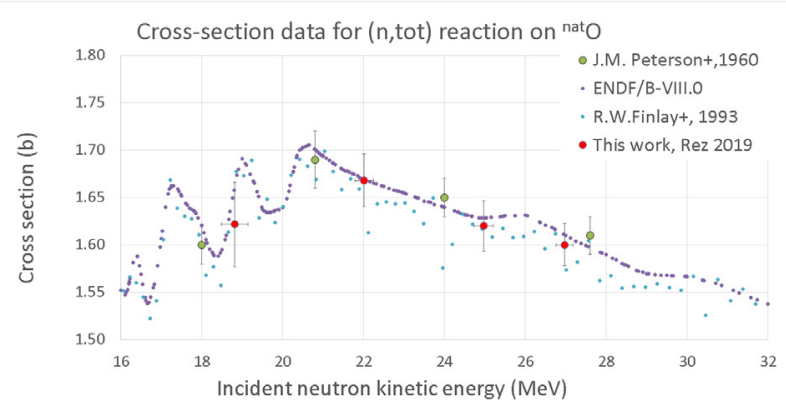

Figure 5. Present data of total neutron cross-section for ${ }^{\text {nat }} \mathrm{O}$ are shown. For comparison data of Peterson [10] and Finlay [11] together with ENDF/B-VIII.0 evaluation for ${ }^{16} \mathrm{O}(\mathrm{n}$, tot) reaction [6] are shown.

\section{Discussion and Conclusion}

The new $1.6 \mathrm{~m}$ long neutron beam collimator was recently constructed at the NPI's U-120M cyclotron facility. The collimator was used during the transmission measurements and contributed significantly to the precision of the final results. Quasi-monoenergetic neutron beams of four different energies were used in combination with the TOF detection technique. The transmission sample consisted of liquid oxygen of natural abundance which was placed into the polystyrene box. Four new data points plotted in Fig. 5 are compared with only two other experimental data sets ([10] and [11]) which were measured with a similar degree of precision at given energy range from 18 to $28 \mathrm{MeV}$. In Fig. 5 data point from ENDF/B-VIII.0 [6] for ${ }^{16} \mathrm{O}$ (n, tot) reaction are plotted as well. The comparison given in Fig. 5 shows very good agreement with both of the previous works as well as with the data points of evaluation. Although the agreement between compared data sets is within the limits defined by standard deviations it can be seen that the above energy of $22 \mathrm{MeV}$ present data are in better agreement with Finlay's work. Overall except the $22 \mathrm{MeV}$ data point present results are slightly below the evaluation. Further investigation of neutron total cross-section on oxygen in energy region $18-35 \mathrm{MeV}$ with white neutron source is planned at the NPI.

\section{Acknowledgments}

Measurements were carried out at the CANAM infrastructure of the NPI CAS Rez supported through MŠMT project No. LM2015056.

This publication was supported by OP RDE, MEYS, Czech Republic under the project CANAM, no. EF16_013/0001812.

Author (M.A.) was supported through project of the MEYS Spiral2-CZ, CZ.02.1.01/0.0/0.0/16_013/0001679.

Access to computing and storage facilities owned by parties and projects contributing to the National Grid Infrastructure MetaCentrum provided under the programme "Projects of Large Research, Development, and Innovations Infrastructures" (CESNET LM2015042), is greatly appreciated.

\section{References}

[1] P. Bém et al., http://dx.doi.org/10.1051/ndata:07598 (2007)

[2] J. Novák et al., EPJ Web of Conferences (2019), paper submitted for publication, ND2019.

[3] M. Majerle, P. Bém, J. Novák, E. Šimečková, M. Štefánik, Nuclear Physics A 953, 139 (2016)

[4] R. Brun, F. Rademakers, Nuclear Instruments and Methods in Physics Research Section A: Accelerators, Spectrometers, Detectors and Associated Equipment 389, 81 (1997)

[5] M. Morháč et al., Nuclear Instruments and Methods in Physics Research Section A: Accelerators, Spectrometers, Detectors and Associated Equipment 401, 385 (1997)

[6] D. Brown et al., Nuclear Data Sheets 148, 1 (2018), special Issue on Nuclear Reaction Data

[7] E. D.B. Pelowitz, LA-CP-11-00438 (2011)

[8] M. Chadwick et al., Nuclear Data Sheets 112, 2887 (2011), special Issue on ENDF/B-VII.1 Library

[9] M. Auman et al., Phys. Rev. C 5, 1 (1972)

[10] J.M. Peterson, A. Bratenahl, J.P. Stoering, Phys. Rev. 120, 521 (1960)

[11] R.W. Finlay et al., Neutron Total Cross Section Measurements at Intermediate Energy, in Nuclear Data for Science and Technology (1992), pp. 720-722, ISBN 978-3-642-58113-7 